\title{
A Competitive Swarm Optimizer-Based Technoeconomic Optimization with Appliance Scheduling in Domestic PV-Battery Hybrid Systems
}

\author{
Bo Wang $\mathbb{D}^{1},{ }^{1}$ Yanjing Li, ${ }^{1}$ Fei Yang, ${ }^{2}$ and Xiaohua $\mathrm{Xia}^{3}$ \\ ${ }^{1}$ Key Laboratory of Ministry of Education for Image Processing and Intelligent Control, \\ Artificial Intelligence and Automation School, Huazhong University of Science and Technology, Wuhan 430074, China \\ ${ }^{2}$ Department of Artificial Intelligence and Automation, School of Electrical Engineering and Automation, Wuhan University, \\ Wuhan 430072, China \\ ${ }^{3}$ Department of Electrical, Electronic and Computer Engineering, University of Pretoria, Pretoria 0028, South Africa \\ Correspondence should be addressed to Bo Wang; wb8517@hust.edu.cn
}

Received 19 July 2019; Accepted 28 August 2019; Published 13 October 2019

Guest Editor: Chun Wei

Copyright (c) 2019 Bo Wang et al. This is an open access article distributed under the Creative Commons Attribution License, which permits unrestricted use, distribution, and reproduction in any medium, provided the original work is properly cited.

\begin{abstract}
A technoeconomic optimization problem for a domestic grid-connected PV-battery hybrid energy system is investigated. It incorporates the appliance time scheduling with appliance-specific power dispatch. The optimization is aimed at minimizing energy cost, maximizing renewable energy penetration, and increasing user satisfaction over a finite horizon. Nonlinear objective functions and constraints, as well as discrete and continuous decision variables, are involved. To solve the proposed mixed-integer nonlinear programming problem at a large scale, a competitive swarm optimizer-based numerical solver is designed and employed. The effectiveness of the proposed approach is verified by simulation results.
\end{abstract}

\section{Introduction}

Making best use of renewable energies has been a topic that receives continuous attention [1]. The photovoltaic (PV) energy is one of the most concerned renewable energy because of the ubiquity of solar irradiation and very low carbon emission [2]. The PV energy generation is therefore integrated into power grids in many countries [3]. However, because of PV energy's intermittent nature, it is difficult to use PV energy alone to support sustained power demands in the complicated context, such as domestic electrical loads. A popular paradigm to utilize the PV power source is to integrate the PV energy into hybrid energy systems, where multiple power sources are adopted and dispatched cooperatively [4]. There were an enormous number of studies on hybrid system optimization over the past twenty years. Advanced technologies have been applied to the economic power dispatch problem in hybrid energy systems [5-7]. Most of such studies focused on power flow control strategies where the demand side was rather considered constraints in the system. There were also studies that introduced demand-side management into hybrid energy system management $[8,9]$, whereas these studies hardly explored the potentials of controlling both power flows and load behaviors. In general, the potentials of incorporating the power dispatch with demand-side management remain to be explored at the current stage. Indeed, such a problem is difficult because of the complex correlations between power sources and loads and its large-scale nature.

In this paper, a technoeconomic optimization problem is extended and improved to investigate further energy efficiency and economic potentials in such a domestic gridconnected PV-battery hybrid energy system, based on a series of previous studies by Tazvinga et al. [10-15]. There are two parts of the interventions: the power dispatch that decides which power is to be supplied to which load and appliance time scheduling that decides when to activate a specific domestic appliance to fulfill the user requirements. 
The optimization is implemented over a finite time horizon. There are three objectives involved in the optimization. Firstly, the overall energy cost over the horizon has to be minimized. The energy cost mainly comes from the consumed grid power, based on the time-of-use (TOU) tariff [16]. The battery wear cost is also integrated into the overall cost. Secondly, the usage of renewable energy has to be maximized. Given that the overall power demand from the loads is considered constant, this objective is transformed into the grid power consumption minimization. Thirdly, the user satisfaction has to be maximized. An inconvenience indicator has been proposed by Setlhaolo and Xia [12] so that the overall difference between the scheduled appliance operation and the baseline appliance operation is calculated. The objective is thus introduced by minimizing such an inconvenience indicator. A weighted sum approach is employed to simultaneously optimize the three objectives, subject to a series of system constraints.

The main contributions of this study are listed as follows: Firstly, the hybrid system design is improved. The major improvement from the design perspective is that separate power dispatch is introduced to each connected appliance, instead of considering the electrical load (consisting of a variety of appliances) as a whole. This is realized by introducing additional power lines between each pair of the appliances and power sources. The power dispatch is thus managed in a more flexible way. In the previous model, all appliances are compelled to choose the same power source at one time. In the improved system design, additional power lines and switches are deployed such that appliances can choose the power sources themselves. The supply that combines various power sources provides the system more flexible power dispatch choices. For example, at peak hours, the battery bank cannot support heavy loads independently because of its capacity limitation. According to the previous design, the battery bank can only work for a short time in the late evening; otherwise, the power demands cannot be matched. In the new design, the battery bank has a much longer possible working time by only supporting a part of the loads, which leaves more space for the load scheduling. Improved flexibility brings more energy efficiency potentials, and thus, more economic benefits can be achieved.

Secondly, the mathematical formulation of the technoeconomic optimization is improved, as the dimension of decision variables is minimized. The aforementioned flexibility improves at the cost of additional decision variables, i.e., the ON/OFF states of the additional switches. The number of decision variables grows largely as the problem scale grows spatially with the number of switches and longitudinally with the number of control intervals, in comparison with the previous system design. To reduce the resulting computational burden, the interplay among the switch behaviors is identified. Some switch behavior constraints are involved; for example, the battery can only charge or discharge at one time, and the appliance can only have one active power line connection. The correlated switches manifest finite states; as a result, a set of discrete state variables are introduced. The values of the discrete variables indicate the combination of ON/OFF states of the correlated switches. Given the constraints, the state variables choose values from a limited range. In this way, the number of variables to describe the complex and interacting switch behaviors is minimized. As the ON/OFF states of the switches are a major part of the decision variables, the dimension of the decision variables is largely reduced; therefore, the computational burden is smaller such that the problem is more promising to be solved within limited time.

Thirdly, an advanced numerical solver is designed for the proposed problem. A major difficulty to implement the technoeconomic optimization is the solver. The investigated problem involves nonlinear objective functions and constraints, as well as continuous and discrete decision variables. It is thus a mixed-integer nonlinear programming (MINLP) problem. Furthermore, as mentioned above, the decision variable can be a large number, e.g., over 700. A proper solver to such a complicated and large-scale optimization problem is thus required. An intelligent optimization algorithm, namely, the competitive swarm optimizer (CSO), is employed to design the numerical solver. Cheng and Jin firstly proposed the CSO algorithm to solve largescale optimization problems [17]. The CSO algorithm is designed on the basis of the particle swarm optimization (PSO) algorithm with a very different searching mechanism. In the PSO algorithm, the term "particle" is employed to refer to the individual solutions. The particles are characterized with two vectors, namely, the position and velocity vectors. The position vector describes the value of a solution and the velocity vector the incremental of the value. The PSO updates the position vector with the velocity vector via interacting with the global best position in the swarm (the population of individuals) and the personal best position in history [18]. The CSO algorithm adopts the position and velocity vector modelling from PSO but employs a random pairwise competition mechanism such that the loser particle can learn from the winner particle to update its position and velocity. In this way, the CSO algorithm can reduce the opportunity of convergence to local optimum, thereby manifesting better and satisfying overall performances than large-scale PSO algorithms. The numerical solver is designed on the pairwise competition concept basis and modified to better match the investigated scenarios.

A case study is employed to test and verify the effectiveness of the proposed approach, where the power dispatch and appliance time scheduling on a daily basis are applied to a typical South African household hybrid system. To thoroughly investigate the effectiveness, results from three cases, where different objective functions are applied with the new flexible power dispatch and the previous dispatch method, are illustrated and analyzed. For all cases, the CSObased numerical solver is employed, and thus, the power dispatch methods are focused and compared.

The remainder of this paper is structured as follows: Section 2 introduces the hybrid system component modelling. Section 3 takes advantage of the component modelling to formulate the technoeconomic optimization problem. Section 4 describes the CSO-based numerical solver. Section 5 shows the case study with simulation results and analysis. Section 6 draws the conclusion. 


\section{Domestic PV-Battery Hybrid System}

A domestic grid-connected PV-battery hybrid system is hereby employed as the investigated hybrid system. The general layout of the PV-battery hybrid system is illustrated in Figure 1. The main purpose of such a system is to supply the daily activities of a number of domestic appliances, e.g., electrical water heater (EWH), stove, television set, and washing machine. The involved appliances are connected to both the power grid and the PV system. A battery bank is also introduced to facilitate the power dispatch. The battery bank is able to charge from the power sources, which in this case are the power grid and PV system, and discharge to supply the appliances. In order to make use of all possible power sources in the system, a power management unit (PMU) is thereby introduced to implement the energy conversion and the power dispatch. In this way, the PMU manages the operation of the system, including (1) the selection of energy flow to support the active appliances, (2) the time scheduling of the appliances, and (3) the energy conversion and voltage/current matching. It is clear that the $\mathrm{PMU}$ is the central piece of the hybrid system that manages from power quality to energy balance. An assumption is employed that the voltage/current matching is well maintained by the PMU. Our investigation focuses on the power scheduling.

The power management diagram is depicted in Figure 2. There are several components in the PMU. From the PV side, there are a solar charge controller and inverter. The charge controller integrates a DC/DC converter to tune the PV output to match the DC loads, including the battery. The inverter receives input from the charge controller and battery and converts the DC power inputs into the AC loads, which in this case refer to the appliances. From the grid side, there is an $\mathrm{AC}$ charger integrating an $\mathrm{AC} / \mathrm{DC}$ converter that allows the battery be charged by the grid. There are also a number of controllable switches to implement switching control strategies. As Figure 2 depicts, there is one switch for each power line. The ON/OFF states of the switches control the power flows in the system, i.e., the pair of the appliance and its supplier. For each connected appliance, there are a set of switches that control whether the appliance is supplied by the PV, the battery, or the power grid. The arrows on each power line indicate the direction of the power flow. As mentioned above, the additional switches are deployed such that any connected appliance can choose among multiple power sources by adjusting the ON/OFF status of switches. Taking advantage of the developing smart grid and smart building technologies, appliances are equipped with open communication interfaces, which allow the PMU to schedule the activities of the appliances and switches simultaneously, in both wired and wireless manners. As a result, the system design in Figure 2 becomes feasible in practice.

Remark 1. There are more and more domestic loads that can be made DC in the modern daily life; for example, an AC light bulb can be replaced with a DC light-emitting diode (LED) bulb. In the future, it is possible to connect

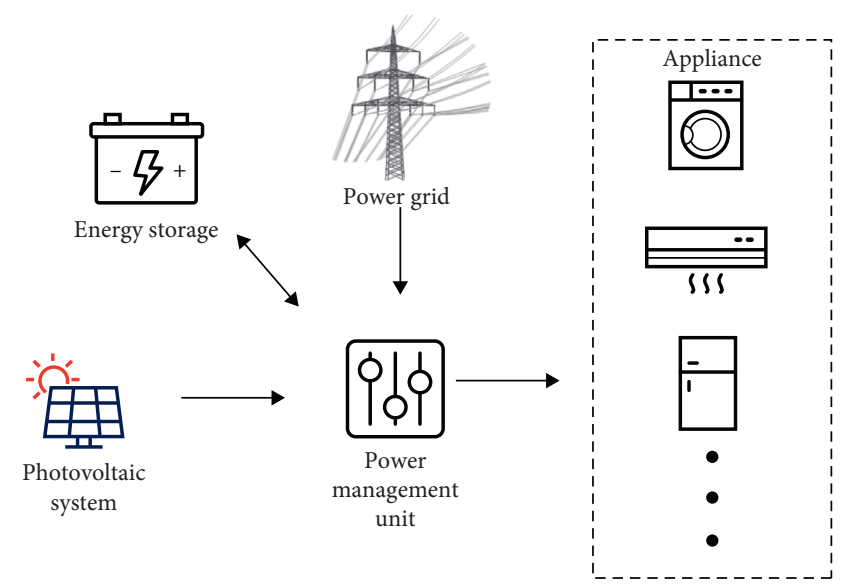

Figure 1: General layout of the hybrid system.

the DC loads directly to the DC power sources, e.g., the PV and battery bank. Such a system may reduce the operational cost of renewable energy resources. However, currently, most domestic appliances are made AC for the sake of standardization. Consequently, the system design mainly focuses on the AC loads at the current stage; therefore, all involved power sources are tuned to be AC suppliers. Direct connections between the DC suppliers and the DC loads can be involved in the future design.

Assume that there are $n$ appliances connected to the system. A set of binary variables are employed to denote the ON/OFF state of the switches. Let $k$ denote the time instant during operation, $g_{1}(k)$ denotes the ON/OFF state of the switch between the PV charge controller and the battery, $g_{2}(k)$ the charge controller and the inverter, $g_{3}(k)$ the AC charger and the battery, and $g_{4}(k)$ the battery and the inverter. $g_{21}(k), g_{22}(k), \ldots, g_{2 n}(k)$ denote the switches between the PV and the appliances, $g_{41}(k), g_{42}(k), \ldots, g_{4 n}(k)$ the battery and the appliances, and $g_{51}(k), g_{52}(k), \ldots, g_{5 n}(k)$ the grid and the appliances. $t$ denotes the time instant over the operation. $P_{1}(k)$ and $P_{4}(k)$ denote the charging power from the PV and the grid, respectively. $P_{2}(k), P_{4}(k)$, and $P_{5}(k)$ denote the power outputs of the respective power sources.

In this system, the operation of appliances is managed together with the power flows. In this study, the operation management is actually time scheduling, as the powers of appliances are considered known a priori and invariable. The time scheduling is implemented simultaneously with the power flow management such that the supply can match the demand and achieve higher energy efficiency potentials.

The mathematical formulations of the behavior for each component in the system are introduced as follows.

2.1. PV Systems. The PV consists of arrays of solar cells such that the solar energy is converted into electrical power. The converted power is proportional to the solar irradiation and the size of PV panels. As an alternative power source to the power grid, the power output is a major concern of the PV system. It is formulated as follows: 


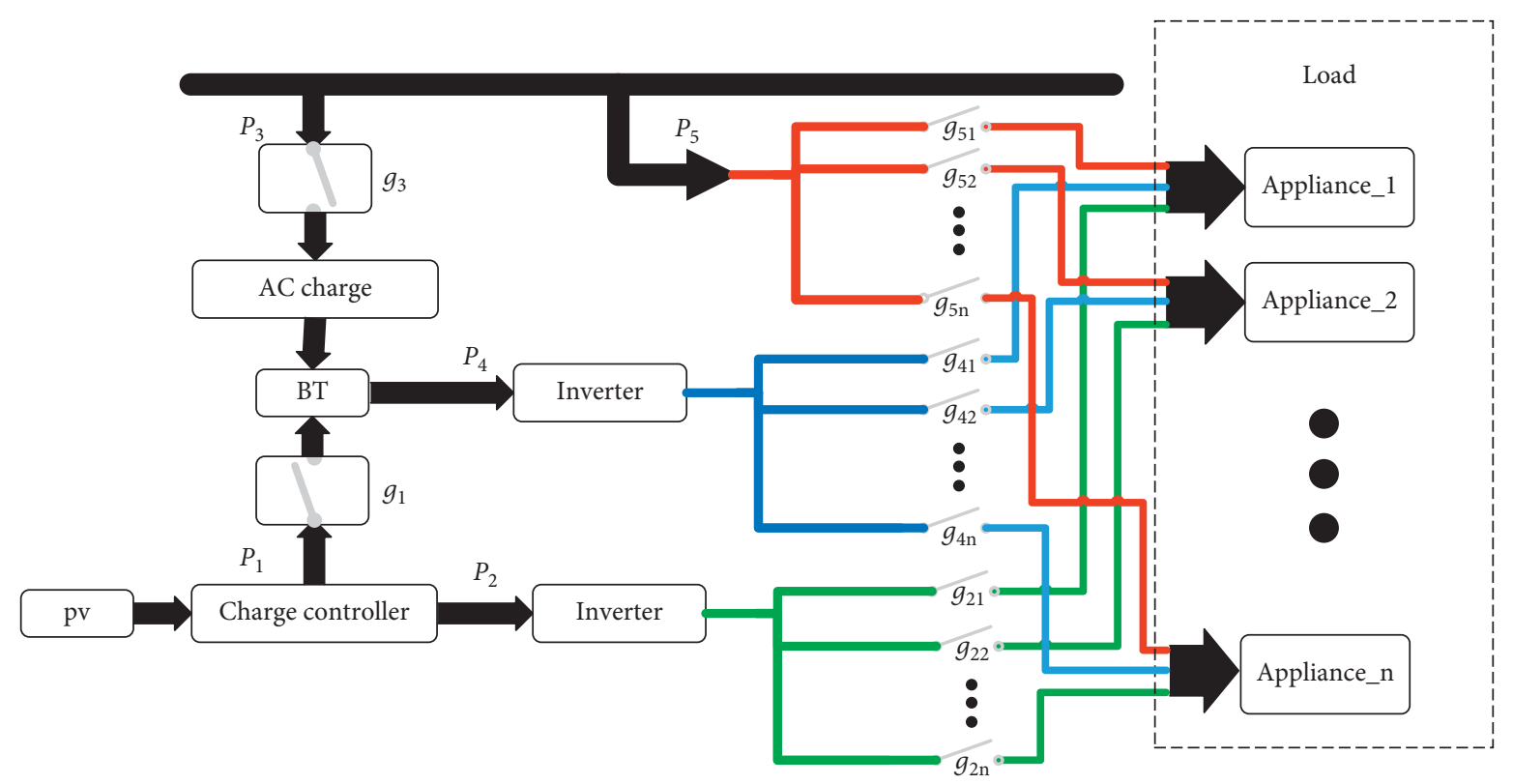

Figure 2: Schematic of the investigated power management system.

$$
P_{p v}=\eta_{p v} I_{p v} A_{c}
$$

where $P_{p v}$ denotes the hourly PV power output $(\mathrm{kW}), \eta_{p v}$ denotes the efficiency of the solar cells, $I_{p v}$ denotes the hourly solar irradiation per unit area $\left(\mathrm{kW} / \mathrm{m}^{2}\right)$, and $A_{c}$ indicates the area of the PV panels that receive solar irradiation $\left(\mathrm{m}^{2}\right)$. There is an intermittent nature of the PV system; that is, $I_{p v}$ can be absent at some sampling instants. An output profile of the PV system is therefore required. Usually, the PV outputs over succeeding 24 hours are predictable [19]. $Q_{p v}$ denotes a time period where $P_{p v}>0$. For $k \notin Q_{p v}, \mathrm{P}_{\mathrm{pv}}$ is considered to be zero given a very small $\mathrm{I}_{\mathrm{pv}}$. A $\mathrm{PV}$ output profile is usually employed to indicate the hourly PV power outputs in a day. $Q_{p v}$ can be identified via the PV output profile.

2.2. Battery Bank. The battery bank charges from other power sources and discharges to support electrical load activities. The battery bank behaviors are dynamic owing to the complicated scheduling of both power sources and appliances. The state of charge (SOC) is employed to characterize the battery bank status. The dynamics of the SOC can be formulated as follows:

$$
\begin{aligned}
\operatorname{Soc}(k+1)= & \operatorname{Soc}(k)+\eta_{B} g_{1}(k) P_{1}(k)+\eta_{B} \eta_{c} g_{3}(k) P_{3}(k) \\
& -g_{4}(k) P_{4}(k),
\end{aligned}
$$

where $\operatorname{Soc}(k)$ denotes the SOC at sampling instant $k ; g_{1}(k)$, $g_{3}(k)$, and $g_{4}(k)$ are binary variables that denote the ON/ OFF state of the respective switches at instant $k$, as depicted in Figure 2; $P_{1}(k), P_{3}(k)$, and $P_{4}(k)$ are the aforementioned power outputs; $\eta_{c}$ denotes the energy conversion efficiencies of the AC charger; and $\eta_{B}$ denotes the battery charging efficiency during operation. Given the current-stage battery system limitations, the simultaneous charging from two different sources or simultaneous charging and discharging are considered unpermitted. A constraint of the switches must be taken into account:

$$
g_{1}(k)+g_{3}(k)+g_{4}(k) \leq 1
$$

such that only one of the switches $g_{1}, g_{3}$, and $g_{4}$ can be turned on at the same time. Following (2), the SOC at a given time $\tau$ can be formulated as follows:

$$
\begin{aligned}
\operatorname{Soc}(\tau)= & \operatorname{Soc}(0)+\eta_{B} \sum_{k=0}^{\tau} g_{1}(k) P_{1}(k)+\eta_{B} \eta_{c} \sum_{k=0}^{\tau} g_{3}(k) P_{3}(k) \\
& -\sum_{k=0}^{\tau} g_{4}(k) P_{4}(k),
\end{aligned}
$$

where $\operatorname{Soc}(0)$ denotes the initial state of the battery bank. $\operatorname{Soc}(k)$ is subject to the following constraint:

$$
C^{\min } \leq \operatorname{Soc}(k) \leq C^{\max }
$$

where $C^{\text {min }}$ and $C^{\text {max }}$ denote the minimum and maximum available capacity $(\mathrm{kWh})$, respectively.

The battery wear level is also evaluated. The wear cost is formulated as follows:

$$
J_{B}(\tau)=\varphi_{b} C_{D}(\tau) \frac{B_{C}}{\mathrm{TH}}
$$

where $C_{D}(\tau)=\sum_{k=0}^{\tau} P_{4}(\tau)$ denotes the overall throughput of the battery bank until instant $\tau$ and $B_{C} / \mathrm{TH}$ denotes the battery wear cost per $1 \mathrm{kWh}$ from throughput energy, in which $B_{C}$ denotes the battery cost and $\mathrm{TH}$ denotes the overall throughput energy. The calculation of $B_{C} / \mathrm{TH}$ can be found from previous studies $[15,20,21]$.

2.3. Power Grid. From the hybrid system viewpoint, the grid supplies infinite and stable electricity at an alternative 
voltage level of $220 \mathrm{~V}$. The grid power comes with a price, which results in the major operational costs. As mentioned above, a TOU tariff is introduced such that the demand response can be implemented. Let $\rho(k)$ denote the TOU electricity price at time $k$ of a day. $\rho(k)$ changes according to which period the time $k$ lies within. The overall operational cost at a given time $\tau$ can be formulated as follows:

$$
\operatorname{Cost}(\tau)=\sum_{k=0}^{\tau} \rho(k)\left[P_{3}(k)+P_{5}(k)\right] .
$$

2.4. Appliances. The electrical loads consist of the load profiles of all appliances connected to the hybrid system. The boundary identification must be conducted before system design. Given the domestic scenario of the investigated system, several reasonable simplifications are made to characterize the demand-side activities. Firstly, all appliances within the system require standard AC power. Secondly, each appliance is subject to a respective constant operation duration. Control strategies can be involved to determine the operation of such appliances $[22,23]$.

According to previous studies [12-14], from the timescheduling perspective, domestic appliances can be categorized into three types: the flexible loads, the shiftable loads, and the fixed loads. The flexible loads' working times can be scheduled freely, at any favorable working time. The shiftable loads' working times can be scheduled within a preferable but limited time period. The fixed loads' working times are fixed, unchangeable in any case. For an arbitrary appliance from any type, the operation duration is constant such that the time scheduling can be characterized as the selection of the starting instant.

In the investigated system, an appliance is connected to the PV, battery bank, and grid. An appliance $i$ from the $n$ appliances is given. As depicted in Figure 2, there is one switch for each power source connection. The ON/OFF states of the three switches at instant $k$ are denoted by $g_{2 i}(k)$, $g_{4 i}(k)$, and $g_{5 i}(k)$. The simultaneous supply from multiple power sources is unpermitted; therefore, a switch constraint is introduced as follows:

$$
g_{2 i}(k)+g_{4 i}(k)+g_{5 i}(k) \leq 1, \quad i=1,2, \ldots, n .
$$

Let $S t_{i}$ denote the starting instant of the appliance $i$ and $D_{i}$ the operation duration. The appliance continuously operates until the end instant, denoted by $E n_{i}=S t_{i}+D_{i}-1$. A continuous operation constraint is introduced as Xia and Zhang proposed [24]:

$$
\begin{aligned}
\sum_{k=0}^{N-D_{i}+1} u_{i}(k) u_{i}(k+1) u_{i}(k+2) \ldots u_{i}\left(k+D_{i}-1\right) & =1, \\
i & =1,2, \ldots, n,
\end{aligned}
$$

where $u_{i}(k)$ is a binary variable that indicates whether the appliance $i$ is active at time $k$, i.e., the time schedule, and $N$ denotes the length of a finite scheduling period. In this case, $u_{i}(k)=1$ if $S t_{i} \leq k \leq E n_{i}$, and $u_{i}(k)=0$ if otherwise. It is notable that the continuous operation constraint is associated with the actual switch behavior in the following way:

$$
\begin{cases}g_{2 i}(k)+g_{4 i}(k)+g_{5 i}(k)=1, & \text { if } u_{i}(k)=1, \\ g_{2 i}(k)+g_{4 i}(k)+g_{5 i}(k)=0, & \text { if } u_{i}(k)=0\end{cases}
$$

Let $\bar{p}_{i}(t)$ with $t=0,1,2, \ldots, D_{i}-1$ denote the load profile over the operation period $\left(0, D_{i}-1\right)$ of the appliance $i$. Assuming that $\bar{p}_{i}(t)$ are known a priori, the power demand $p_{i}(k)$ can thus be determined: if $k=S i+t, p_{i}(k)=\bar{p}_{i}(t)$; otherwise, $p_{i}(k)=0$.

2.5. System Constraints. A series of constraints must be introduced to the system such that the operation requirements are all satisfied and none of the physical laws is violated.

(a) The energy balance must be fulfilled anytime during operation, as indicated by the following equation:

$$
\left[\begin{array}{c}
\eta_{I 2} P_{2}(k) \\
\eta_{I 4} P_{4}(k) \\
P_{5}(k)
\end{array}\right]=\sum_{i=1}^{n}\left[\begin{array}{c}
g_{2 i}(k) \\
g_{4 i}(k) \\
g_{5 i}(k)
\end{array}\right] p_{i}(k),
$$

where $P_{2}(k), P_{4}(k)$, and $P_{5}(k)$ are the power flows from the PV output, battery bank discharge, and power grid supply and $\eta_{I 2}$ and $\eta_{I 4}$ denote the inverter efficiency of the PV and battery bank, respectively. For the convenience of calculation, a logical state variable $s w_{i}(k)$ is employed to describe the combination of $g_{2 i}(k), g_{4 i}(k)$, and $g_{5 i}(k) . s w_{i}(k)$ chooses a value from $\{0,1,2,3\} . s w_{i}(k)=0$ indicates the state that all switches are turned off and the switch states equal to $0 . s w_{i}(k)=1$ indicates that only $g_{2 i}(k)=1, s w_{i}(k)=2$ that only $g_{4 i}(k)=1$, and $s w_{i}(k)=3$ that only $g_{5 i}(k)=1$.

(b) The capacity constraints must be followed such that the power flow is kept within the range of the component capacity. The power flows in the system are thus limited. The power flows $P_{1}(k)$ and $P_{2}(k)$ are subject to the following constraint:

$$
0 \leq P_{1}(k)+P_{2}(k) \leq \eta_{s} P_{p v}(k),
$$

where $P_{p v}(k)$, as mentioned above, is the PV output at time $k$ and $\eta_{s}$ denotes the charge controller efficiency. The power flows $P_{2}(k)$ and $P_{4}(k)$ as the supplier are subject to the following constraints:

$$
\left\{\begin{array}{l}
0 \leq \eta_{I 2} P_{2}(k) \leq P_{I 2}(k), \\
0 \leq \eta_{I 4} P_{2}(k) \leq P_{I 4}(k),
\end{array}\right.
$$


where $P_{I 2}(k)$ and $P_{I 4}(k)$ denote the inverter capacity of the PV and battery bank, respectively. The power flows $P_{3}(k)$ and $P_{5}(k)$ are subject to the following constraint:

$$
0 \leq g_{3}(k) P_{3}(k)+P_{5}(k) \leq P_{G}^{\max },
$$

where $P_{G}^{\max }$ denotes the allocated grid maximum power for this grid-connected system. The power flows $P_{2}(k), P_{4}(k)$, and $P_{5}(k)$ are obtained from the energy balance equation (11). $P_{1}(k)$ and $P_{3}(k)$ are decided by the scheduling algorithm, and they are part of the decision variables.

(c) The switch control strategies are also subject to constraints that prevent infeasible switch behaviors. These constraints are formulated in preceding sections along with the system component modelling, i.e., constraints (3) and (8)-(10). Some further associations of the switch behaviors are identified as follows:

$$
\begin{gathered}
\begin{cases}g_{1}(k)=1, & \text { if } P_{1}(k)>0, \\
g_{1}(k)=0, & \text { if } P_{1}(k)=0, \\
g_{3}(k)=1, & \text { if } P_{3}(k)>0, \\
g_{3}(k)=0, & \text { if } P_{3}(k)>0,\end{cases} \\
\left\{\begin{array}{l}
g_{2}(k)=1, \quad \text { if } \prod_{i=1}^{n}\left(\operatorname{sw}_{i}(k)-1\right)=0, \\
g_{2}(k)=0, \quad \text { if otherwise, } \\
g_{4}(k)=1, \quad \text { if } \prod_{i=1}^{n}\left(\text { sw }_{i}(k)-2\right)=0, \\
g_{4}(k)=0, \quad \text { if otherwise. }
\end{array}\right.
\end{gathered}
$$

In this way, the states of $g_{1}(k)-g_{4}(k)$ at time $k$ can be identified from the values of $P_{1}(k), P_{3}(k)$, and $\mathrm{sw}_{i}(k)$ with $i=1,2, \ldots, n$.

\section{Problem Statement}

The primary management objective of the investigated optimization problem is cost minimization. Secondly, the renewable energy penetration is involved; that is, the usage of grid power should be minimized as well. Owing to the TOU tariff, the two objectives manifest certain differences and must be equally considered in scheduling. Furthermore, the user satisfaction in time scheduling is taken into account. All these objectives are evaluated on a finite horizon basis. Let $T$ denote the number of sampling instants. The optimization problem is formulated as follows.

3.1. Decision Variables. The involved decision variables consist of three parts: (1) the switch control strategy decision variables, (2) the charging power control variables, and (3) the appliance time-scheduling decision variables. It is given that $i=1,2, \ldots, n$ in the following discussion.
The switch control variables are $\mathrm{sw}_{i}(k)$, which can characterize the status of most switches in the system as constraints (15) and (16) imply. Given constraints (8)-(10), it is unnecessary to cover the whole finite horizon. For the appliance $i, \operatorname{sw}_{i}(k)>0$ when $S t_{i} \leq k \leq E n_{i}$, and $\mathrm{sw}_{i}(k)=0$ if $k$ lies outside the working period. Therefore, the minimum required control variables are $\mathrm{sw}_{i}(k)$ with $k \in\left[S t_{i}, E n_{i}\right]$. The dimension of this part is $\sum_{i=1}^{n} D_{i}$.

The charging power control variables are $P_{1}(k)$ and $P_{3}(k)$. Given constraints (3) and (15), $P_{1}(k) * P_{3}(k)=0$ at any given instant $k$; therefore, the dimension of this part is $T$.

As mentioned above, the appliance time scheduling is simplified with the known a priori and constant load profile $\bar{p}_{i}(t)$ and operation duration $D_{i}$. Taking advantage of the knowledge, the scheduling is implemented with decision variables $S t_{i}$. The dimension of this part is $n$.

The dimension of the optimization is therefore $\sum_{i=1}^{n} D_{i}+T+n$. Comparing with the previous study [15], the problem is extended to a higher dimension but simplified by taking advantage of $s_{i}(k)$ and the constraints such that the dimension of the optimization grows slower than the problem scale.

3.2. Objectives. The cost minimization objective is formulated as follows:

$$
J_{c}=\sum_{k=0}^{T} \rho(k)\left[P_{3}(k)+P_{5}(k)\right]+\varphi_{b} J_{B}(T),
$$

where $\varphi_{b}$ denotes a weight that indicates the preferable importance of the battery wearout to the decision-maker.

The renewable energy penetration objective is formulated as follows:

$$
J_{e}=\sum_{k=0}^{T}\left[P_{3}(k)+P_{5}(k)\right] .
$$

The user satisfaction is evaluated via an inconvenience indicator, which is adopted from the study of Setlhaolo and Xia [12]:

$$
\beta=\sum_{i=1}^{n} \gamma_{i} \sum_{k=0}^{T}\left[u_{i}^{b l}(k)-u_{i}(k)\right]^{2},
$$

where $\gamma_{i}$ denotes an importance factor of the appliance $i$ and $u_{i}^{b l}(k)$ denotes the baseline time schedule of the appliance $i$. (19) quantifies the difference between the baseline time schedule and the adopted time schedule. Given the system modelling and constraints in this study, the user satisfaction evaluation is simplified as follows:

$$
\beta=\sqrt{\sum_{i=1}^{n} \gamma_{i}\left[S t_{i}^{b l}-S t_{i}\right]^{2}},
$$

where $S t_{i}^{b l}$ denotes the baseline starting instant. Such a difference has to be minimized such that the user remains happy with the optimized appliance time schedule. 
3.3. Technoeconomic Optimization. Taking advantage of the preceding objective functions and constraint formulations, the technoeconomic optimization problem is obtained by minimizing the following objective function:

$$
J\left(\mathrm{sw}_{i}(k), P_{1}(k), P_{3}(k), S t_{i}\right)=\lambda_{c} J_{c}+\lambda_{e} J_{e}+\lambda_{b} \beta,
$$

subject to the battery dynamics (2) and constraints (3), (8)-(10), and (11)-(16).

According to the above formulations, there are nonlinear objective functions and constraints, as well as continuous and discrete decision variables in the problem. They result in a mixed-integer nonlinear programming (MINLP) problem, at a relatively large scale. The general theoretic approach of solving an MINLP problem remains an open question; as a result, numerical solvers are widely employed. In the previous study [15], an OTPI toolbox https://www.inverseproblem.co.nz/ OPTI/index.php/DL/DownloadOPTI/ in MATLAB was adopted as the numerical solver. The former solver took quite a large amount of time for calculation. In this study, the implementation of intelligent optimization algorithms on such a problem is investigated. A newly proposed algorithm named the competitive swarm optimizer (CSO) is adopted as the numerical solver. The introduction to the CSO-based solver comes in the following section.

\section{Numerical Solver Design}

4.1. Competitive Swarm Optimizer. In the CSO algorithm, let $x$ denote a particle and $w$ and $l$ the indices of the winner and loser particles in a pair. Assume that it is the $G$-th iteration, and there have been $k-1$ competitions. After the $k$-th competition, the next-generation winner particle $x_{w, k}(G+$ $1)$ remains the same as $x_{w, k}(G)$. The loser particle $x_{l, k}(G+1)$, namely, the position vector, is thereby updated as follows:

$$
x_{l, k}(G+1)=x_{l, k}(G)+V_{l, k}(G+1),
$$

where $V_{l, k}(G+1)$ is the next-generation velocity vector, updated as follows:

$$
\begin{aligned}
V_{l, k}(G+1)= & r n_{1}(k, G) V_{l, k}(G)+r n_{2}(k, G)\left[x_{w, k}(G)-x_{l, k}(G)\right] \\
& +\varphi r n_{3}\left[\bar{x}_{k}(G)-x_{l, k}(G)\right]
\end{aligned}
$$

where $r n_{1}, r n_{2}$, and $r n_{3}$ are random vectors; $\bar{x}_{k}(G)$ is the center of neighborhood filed particles, i.e., a set of particles that are close enough to $x_{l, k}(G)$; and $\varphi$ is the weighting coefficient of $\bar{x}_{k}(G)$. Such a neighborhood field is predefined. There is a special case that the neighborhood covers the whole swarm, where $\bar{x}_{k}(G)$ indicates the global mean position of the particles at iteration $G$. The velocity and position vectors are employed for the continuous cases. In a discrete case, e.g., the decision variables $\operatorname{sw}_{i}(k)$ in this study, other update mechanisms must be employed. A crossover mechanism is hereby employed as follows:

$$
\left\{\begin{array}{l}
x_{l, k}(r n, G+1)=x_{w, k}(r n, G), \\
x_{l, k}(\overline{r n}, G+1)=x_{l, k}(\overline{r n}, G),
\end{array}\right.
$$

where $r n$ denotes randomly generated indices of the components in a particle $x$ and $\frac{g}{r n}$ the unselected indices. (24) suggests that the winner particle $x_{w, k}(G)$ selects and copies a part of its components into the next-generation loser particle $x_{l, k}(G+1)$. The unselected components of $x_{l, k}(G+1)$ remain the same as $x_{l, k}(G)$. In this way, the loser particle can learn from the winner particle.

For an MINLP problem, there are simultaneously continuous and discrete components in a particle. In this case, the continuous part and discrete part are separated, (23) and (22) are implemented on the continuous part, and (24) is implemented on the discrete part. After the learning process, the two updated parts are combined again to obtain the particle of next generation. The theoretical proof of the convergence of the CSO algorithm can be referred to [17].

The pseudocode of the CSO according to the preceding introduction is illustrated in Algorithm 1 [25].

Remark 2. The introduced CSO algorithm is mainly designed for continuous problems. For discrete decision variables, e.g., binary variables or integers, the discrete PSO algorithm [26, 27] can facilitate the algorithm design. The pairwise competition can be further introduced to other evolutionary algorithms, such as differential evolution (DE).

Remark 3. Given the investigated MINLP problem (21), the original CSO algorithm cannot be applied in a straightforward way. Modifications that match the decision variables are to be introduced such that satisfying performances can be achieved.

4.2. Modified CSO-Based Solver. In order to implement the $\mathrm{CSO}$ algorithm on a constrained problem, a penalty function is introduced to the original objective function (17). Given that there are $N_{C}$ constraints to a problem, the penalty function is formulated as follows:

$$
P_{\mathrm{en}}=\sum_{k=0}^{T} \sum_{i=0}^{N_{C}} \omega_{\mathrm{Pen}, i} P_{\mathrm{en}, i}(k),
$$

where

$$
P_{\mathrm{en}, i}(k)= \begin{cases}0, & \text { if constraint } i \text { is obeyed, } \\ M, & \text { if constraint } i \text { is violated, }\end{cases}
$$

where $M$ is a large positive number and $\omega_{\text {Pen, } i}$ is the weighting factor for constraint $i$. A fitness function is thereby formulated with (17), (25), and (26):

$$
f(x)=J_{c}+P_{\mathrm{en}} .
$$

In this way, for a minimization problem, a particle becomes much less competitive when any of the constraints 
Definition:

$x$ : the particle;

$P$ : the swarm;

$n p$ : the swarm size, i.e., the number of particles;

$G$ : number of iterations;

$w$ and $l$ : the indices of winner and loser particles;

$f(\cdot)$ : the fitness function, assuming that this is a minimization problem;

Terminal condition: the maximum number of iteration $\mathrm{Mg}$ is reached;

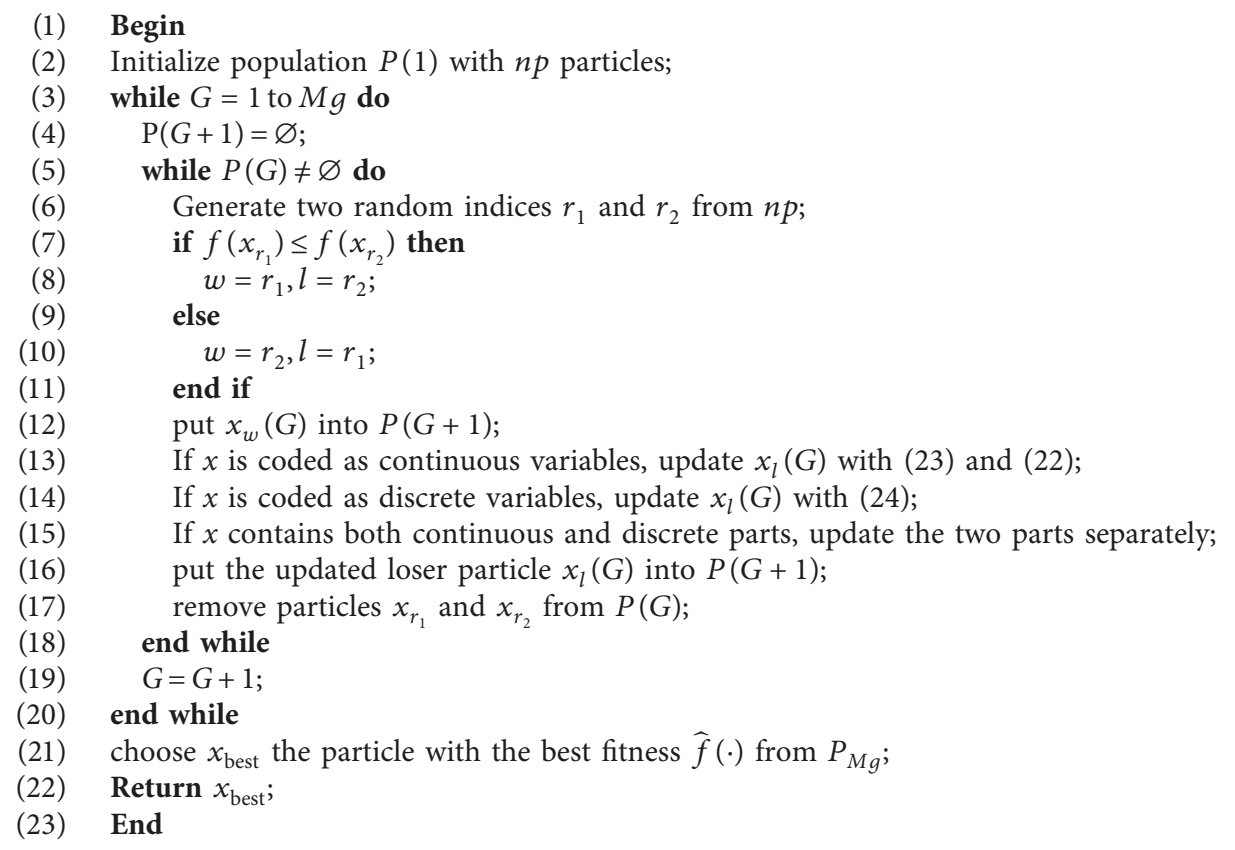

Algorithm 1: Pseudo code of the CSO algorithm.

is violated, given that a large positive will be added to the objective function.

After competition, the loser particle must learn from the winner particle. However, the MINLP search space and constraints in the investigated problem are quite complicated. The dynamics of the battery bank charging and discharging invoke further difficulty to search for the optimum. Consequently, the performance of Algorithm 1 is not satisfying. The following modifications are thereby employed to improve the performances on this specific problem.

Firstly, the learning strategies are modified to improve the searching efficiency. Let $\bar{f}(G)$ denote the mean fitness of the current swarm (at the $G$-th iteration). After competition, the fitness of both winner and loser particles is evaluated:

(i) If $f\left(x_{w, k}(G)\right) \geq \bar{f}(G)$, both winner and loser particles are considered inferior; therefore, the winner particle must learn from the global best particle $x_{\text {gbest }}(G)$, while the loser particle must learn from the winner.

(ii) If $f\left(x_{l, k}(G)\right) \leq \bar{f}(G)$, both winner and loser particles are considered superior; therefore, the winner particle is moved into $P(G+1)$, and the loser particle implements mutation subject to the genetic algorithm style. (iii) If $f\left(x_{w, k}(G)\right) \leq \bar{f}(G)<f\left(x_{l, k}(G)\right)$, the original CSO learning strategies are applied.

Secondly, constraints (3) and (15) are employed to generate battery bank charging and discharging states, namely, the "knowledge-guided solution filter". $\forall k, g_{4}(k)$ is firstly identified subject to (16). If $g_{4}(k)=1$, then $P_{1}(k)=P_{3}(k)=0$. If $g_{4}(k)=0$, then $P_{1}(k)$ is randomly generated within $\left[0, P_{p v}(k)-P_{2}(k)\right)$. If $g_{4}(k)=0$ and $P_{1}(k)=0$, then $P_{3}(k)$ is randomly generated. If the $\operatorname{Soc}(k)$ reaches its upper bound at time $k$, then $P_{1}(k)=P_{3}(k)=0$. In this way, the charging and discharging decision variables are guaranteed to be feasible. The knowledge-guided solution filter reduces cost of trial and error during optimization such that the algorithm can persist searching within a feasible space.

Remark 4. According to simulations, the modified solver constantly outperforms the original CSO algorithm on the investigated problem. There lacks a theoretical analysis on the performances, whereas a hypothesis is made that the superior performances are resulted from the knowledgeguided solution filter. Wang and Zheng [28] reported that exploitation of the algorithm is enhanced by knowledgebased local search. Further details and investigations are expected in future works. 
TABLE 1: Typical usage profiles and baseline time schedules.

\begin{tabular}{|c|c|c|c|c|c|}
\hline \multirow{2}{*}{$\begin{array}{l}\text { Appliances } \\
\text { Index } i\end{array}$} & \multirow{2}{*}{ Power $(\mathrm{kW})$} & \multirow{2}{*}{ Duration (min) } & \multicolumn{2}{|c|}{ Baseline } & \multirow{2}{*}{ Preferable range of $S t$} \\
\hline & & & $S t_{i}$ & $E n_{i}$ & \\
\hline \multicolumn{6}{|l|}{ Shiftable } \\
\hline (1) EWH & 3.0 & $\begin{array}{l}120 \\
120 \\
\end{array}$ & $\begin{array}{c}31 \\
104\end{array}$ & $\begin{array}{c}42 \\
115\end{array}$ & $\begin{array}{c}{[19,31]} \\
{[91,121]}\end{array}$ \\
\hline (2) Stove & 2.5 & $\begin{array}{l}30 \\
50\end{array}$ & $\begin{array}{c}32 \\
113 \\
\end{array}$ & $\begin{array}{c}34 \\
117 \\
\end{array}$ & $\begin{array}{c}{[25,55]} \\
{[97,127]}\end{array}$ \\
\hline $\begin{array}{l}\text { (3) Washing machine } \\
\text { (4) Electric dryer }\end{array}$ & $\begin{array}{l}0.5 \\
2.0\end{array}$ & $\begin{array}{l}60 \\
30\end{array}$ & $\begin{array}{l}109 \\
116\end{array}$ & $\begin{array}{l}114 \\
118\end{array}$ & $\begin{array}{l}{[43,133]} \\
{[49,139]}\end{array}$ \\
\hline $\begin{array}{l}\text { Fixed } \\
\text { (5) Refrigerator } \\
\text { (6) Television set } \\
\end{array}$ & $\begin{array}{l}0.1 \\
0.2\end{array}$ & $\begin{array}{c}1440 \\
180\end{array}$ & $\begin{array}{c}1 \\
104\end{array}$ & $\begin{array}{l}144 \\
121\end{array}$ & $\begin{array}{l}\mathrm{N} / \mathrm{A} \\
\mathrm{N} / \mathrm{A}\end{array}$ \\
\hline $\begin{array}{l}\text { Flexible } \\
\text { (7) Dishwasher } \\
\text { (8) Bread maker } \\
\end{array}$ & $\begin{array}{l}1.8 \\
1.5 \\
\end{array}$ & $\begin{array}{l}150 \\
150 \\
\end{array}$ & $\begin{array}{l}116 \\
118 \\
\end{array}$ & $\begin{array}{l}130 \\
132 \\
\end{array}$ & $\begin{array}{l}{[1,130]} \\
{[1,130]}\end{array}$ \\
\hline
\end{tabular}

\section{Simulation Results and Analysis}

5.1. Case Study. The case study investigates the operation of a household, grid-connected, PV-battery hybrid energy system. The data are retrieved from the South African domestic appliance operation studies [13-15]. There are eight appliances connected in the system. The usage profile of the appliances is shown in Table 1 on a daily basis, where there are 144 time slots, a.k.a., sampling instants. Each time slot lasts 10 minutes. The scheduling horizon is 24 hours, i.e., a whole day. The adopted usage profile and baseline time schedule are for the working day scheduling for a typical South African home. To emphasize, the power of the appliances is measured average power. The baseline time schedule reflects the preferable time according to the inhabitant's habits. For example, the inhabitant turns on the electrical water heater (EWH) twice a day for the hot water demand. In the morning, the EWH is turned on at 5:00 am (the 31-st time slot), operates for two hours, and is turned off at 7:00 am (the 42-nd time slot) such that the user can use heated water after breakfast. In the afternoon, the EWH is turned on again at $5: 10 \mathrm{pm}$ (the 104-th time slot) and turned off at $7: 10 \mathrm{pm}$ (the 115-th time slot) such that the hot water for the evening can be ready. This is the most convenient EWH operation plan for the user. Similarly, the stove must be turn on twice for the cooking demands. The other appliances have to be turned on and off only once daily.

There are several further constraints with the given scenario. Firstly, for the shiftable appliances, a preferable range of starting time slots are given in Table 1 . The flexible appliance can start anytime in a day, and the only requirement is that the operation must be finished before the end of the horizon. The fixed appliance cannot be scheduled; therefore, the preferable range is not applicable (N/A). Secondly, the washing machine and electrical dryer work in a sequence; that is, the dryer must start after the washing machine job is finished. In this case, it results in an additional constraint to the preceding ones:

$$
S t_{4} \geq S t_{3}+D_{3} .
$$

TABLE 2: PV system and battery bank parameters.

\begin{tabular}{lc}
\hline PV capacity $P_{p v}$ & $3.5 \mathrm{~kW}$ \\
Battery bank maximum capacity $C^{\max }$ & $5.04 \mathrm{kWh}$ \\
Battery bank minimum capacity $C^{\min }$ & $2.52 \mathrm{kWh}$ \\
Battery bank cost (ZAR) & $\mathrm{R} 5826$ \\
Initial state of the battery bank & $60 \% \mathrm{C}^{\max }$ \\
AC charger efficiency $\eta_{c}$ & $85 \%$ \\
PV charge controller efficiency $\eta_{s}$ & $90 \%$ \\
PV inverter efficiency $\eta_{I 2}$ & $95 \%$ \\
Battery bank inverter efficiency $\eta_{I 4}$ & $95 \%$ \\
Battery bank charging efficiency $\eta_{B}$ & $80 \%$ \\
\hline
\end{tabular}

TABle 3: Hourly PV output.

\begin{tabular}{lcccc}
\hline Time slot $k$ & {$[0,39)$} & {$[39,45)$} & {$[45,51)$} & {$[51,57)$} \\
$P_{p v}(k)(\mathrm{kWh})$ & 0 & 0.15 & 0.85 & 1.65 \\
\hline$[57,63)$ & {$[63,69)$} & {$[69,75)$} & {$[75,81)$} & {$[81,87)$} \\
2.35 & 2.9 & 3 & 2.95 & 2.55 \\
\hline$[87,93)$ & {$[93,99)$} & {$[99,105)$} & {$[105,111)$} & {$[111,144)$} \\
2 & 1.45 & 0.75 & 0.1 & 0 \\
\hline
\end{tabular}

The adopted PV system and battery bank have their own limitations, as shown in Table 2. The PV system integrates 14 solar panels with the rated power of $0.25 \mathrm{~kW}$. Therefore, the overall capacity, i.e., the rated output, of the $\mathrm{PV}$ system is $3.5 \mathrm{~kW}$. Actually, the PV system output at any given time slot depends on the solar irradiation profile. Such a profile is possible to forecast 24 hours ahead of the scheduling [19]. In the case study, the timely PV output is identified based on an hourly profile in [29], as shown in Table 3, where $Q_{p v}$ is $k \in[39,144)$. The battery bank consists of 4 lead-acid batteries, each with $12 \mathrm{~V}$ rated voltage and $105 \mathrm{Ah}$ rated capacity; that is, the overall capacity is $5.04 \mathrm{kWh}$. The battery cost is calculated in South African rand (ZAR), which is R5826. The lifespan of the battery bank is 1000 cycles at $50 \%$ depth of discharge (http://www.trojanbattery.com/markets/renewableenergy-re/); therefore, the wear cost per $1 \mathrm{kWh}$ throughput energy is $5826 /(1000 * 0.5 * 5.04)=2.312 \mathrm{R} / \mathrm{kWh} . \varphi_{b}=0.1$ 
TABLE 4: TOU tariff.

\begin{tabular}{|c|c|c|}
\hline Time periods & Electricity price & Hours \\
\hline Peak hours & $\mathrm{R} 2.2225 / \mathrm{kWh}$ & {$[08: 00,11: 00) \cup[19: 00,21: 00)$} \\
\hline Standard hours & $\mathrm{R} 0.6773 / \mathrm{kWh}$ & {$[07: 00,08: 00) \cup[11: 00,19: 00) \cup[21: 00,23: 00)$} \\
\hline Off-peak hours & R0.3656/kWh & {$[00: 00,07: 00) \cup[23: 00,24: 00)$} \\
\hline
\end{tabular}

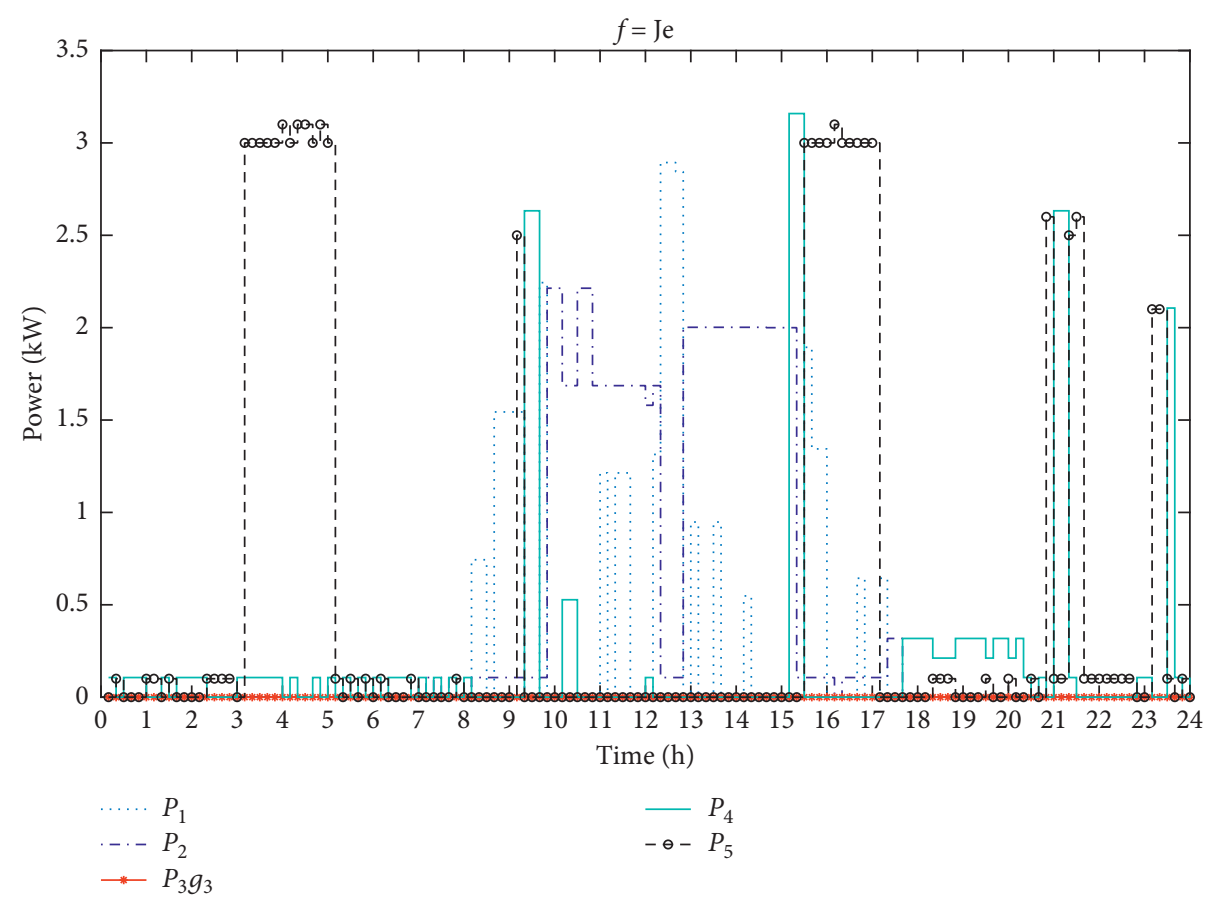

FIgure 3: Power dispatch of the new design in case (i).

such that the usage of the battery is encouraged. The importance factors $\gamma_{i}=1$ for each $i$, i.e., involved appliances, are considered equally important in this case. The efficiencies of the charge controller, AC charger, and inverters are given as well.

The power grid supply is described as follows: The maximum household current is $60 \mathrm{~A}$, which is limited by the utility company. The charging power from the grid is considered constant in this case, which is $5 \mathrm{~kW}$; that is, $P_{3}(k)$ can only be 0 or $5 \mathrm{kWh}$. Furthermore, the TOU tariff is adopted from the study in [13], as shown in Table 4 .

5.2. Simulations. Simulations are programmed in $\mathrm{C}++$ with the following running environment: the CPU is Inter Core i3-8100 CPU@3.60 GHz, the RAM is $16 \mathrm{~GB}$, and the system is Windows $10 \times 64$. Three cases are adopted by adjusting the weighting factors in (21).

(i) $\lambda_{e}=1, \lambda_{c}=0$, and $\lambda_{b}=0$ such that the optimization employs a single objective, i.e., the renewable energy penetration.

(ii) $\lambda_{e}=0, \lambda_{c}=1$, and $\lambda_{b}=0$ such that only the cost minimization objective is optimized.

(iii) $\lambda_{e}=1, \lambda_{c}=1$, and $\lambda_{b}=1$ such that the multiobjective optimization of (21) is implemented where $J_{c}, J_{e}$, and $\beta$ are equally considered.
In each case, there are two demonstrated results. One result is from the proposed approach, and the other one is from the previous power dispatch model [15] as the comparative results. Both results are reported from the average of 20 runs, taking advantage of the proposed CSO-based optimizer. In the CSO algorithm, the swarm size is 1500 and the iteration number is 10000 . The neighborhood field is defined to be the nearest superior particle and inferior particle. The details of such a neighborhood field can be referred to [30, 31].

5.3. Results and Analysis. The results are reported as follows:

(i) In the first case, the grid power supply is minimized to be $14 \mathrm{kWh}$, at the energy cost of R16.57 and inconvenience indicator of 14.25. The power dispatch is depicted in Figure 3. In the comparative result, the minimal grid power is $15.07 \mathrm{kWh}$, at the energy cost of R14.29 and inconvenience indicator of 14.59. The power dispatch is depicted in Figure 4. The improvement of the objective is $7.1 \%$. The average running time is 18.43 minutes.

(ii) In the second case, the cost is minimized to be R7.06, while the overall power supply from the grid is $14.37 \mathrm{kWh}$. The inconvenience indicator is 13.89 . The power dispatch is depicted in Figure 5. In the comparative result, the minimal cost is R7.72, with 


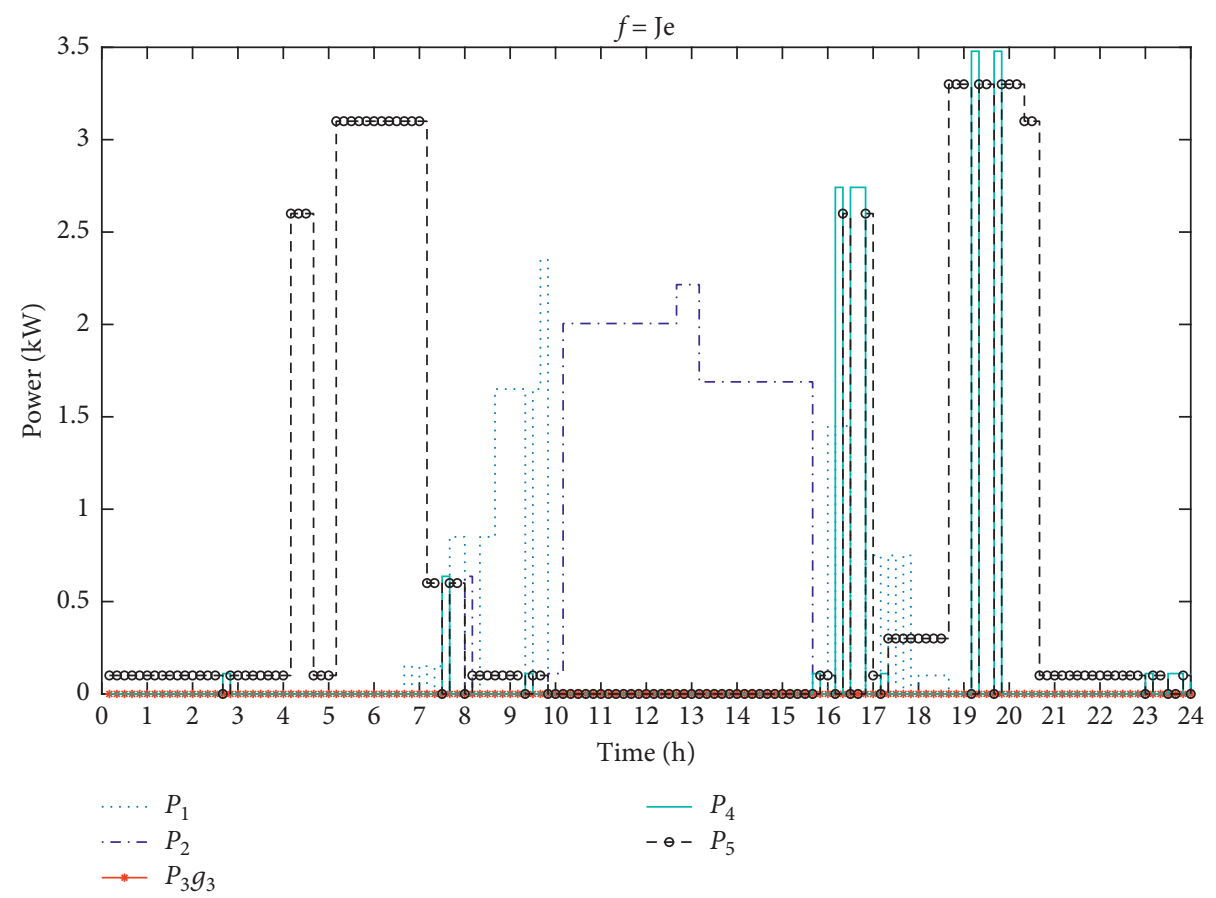

FIgURE 4: Power dispatch of the previous design in case (i).

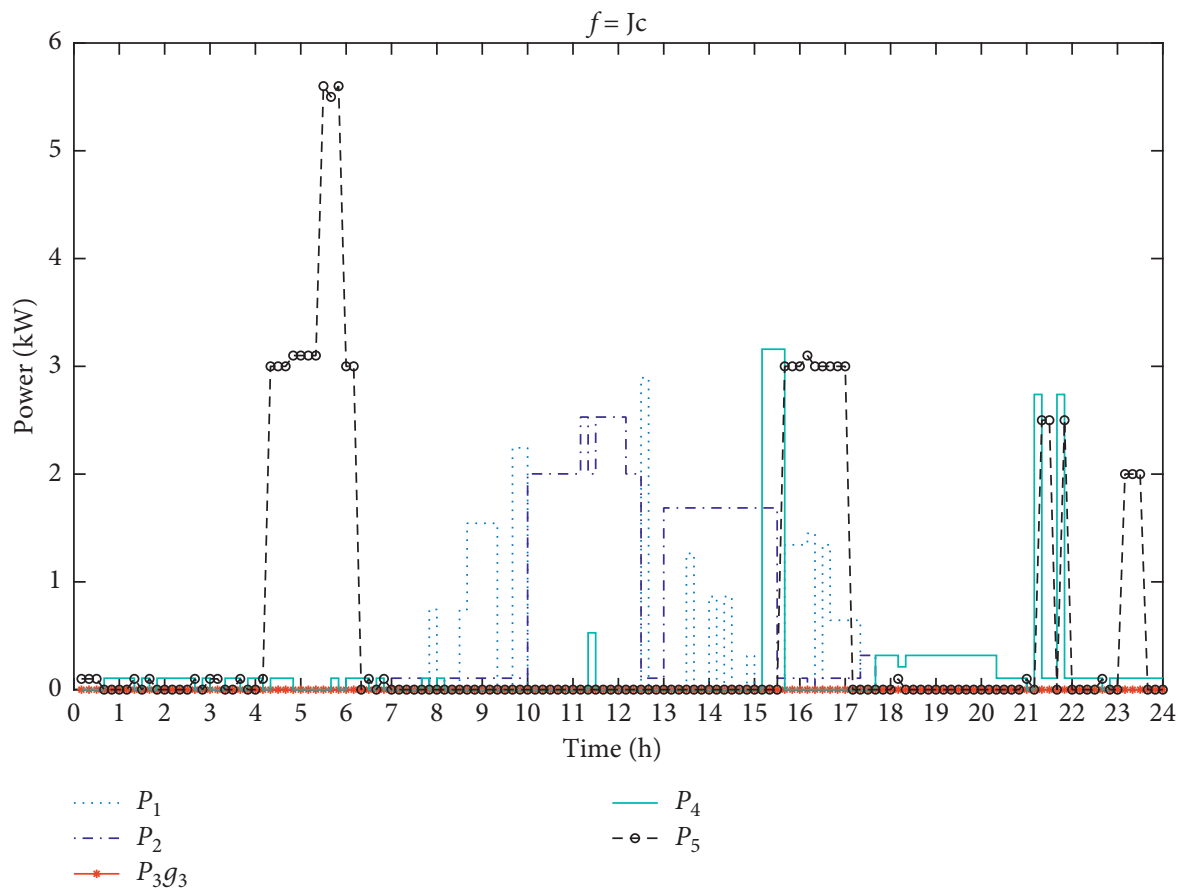

Figure 5: Power dispatch of the new design in case (ii).

the grid power supply of $15.57 \mathrm{kWh}$ and inconvenience indicator of 15.03 . The power dispatch is depicted in Figure 6. The improvement of the objective is $8.6 \%$. The average running time is 18.36 minutes.

(iii) In the third case, the weighted sum of all objectives is minimized. The optimized objective function value is 34.64 , when the energy cost is R8.29 and the grid power supply is $15.53 \mathrm{kWh}$, and the inconvenience indicator is 10.82. The power dispatch is depicted in Figure 7 . In the comparative result, the objective function value is 39.97, where the energy cost is 9.53 and grid power is $18.78 \mathrm{kWh}$, with the inconvenience indicator of 11.66 as well. The power dispatch is depicted in Figure 8. The improvement of the objective is $13.3 \%$. The average running time is 18.37 minutes. 


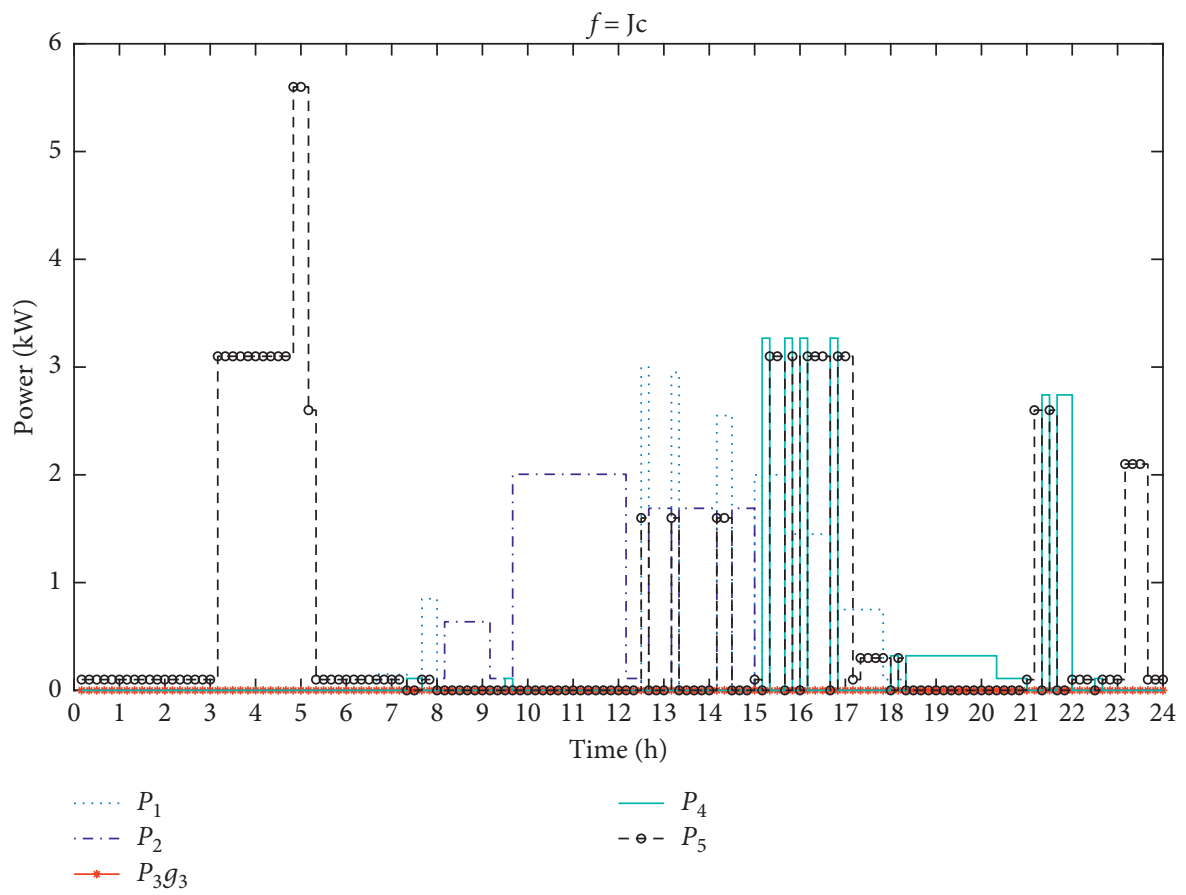

FIgure 6: Power dispatch of the previous design in case (ii).

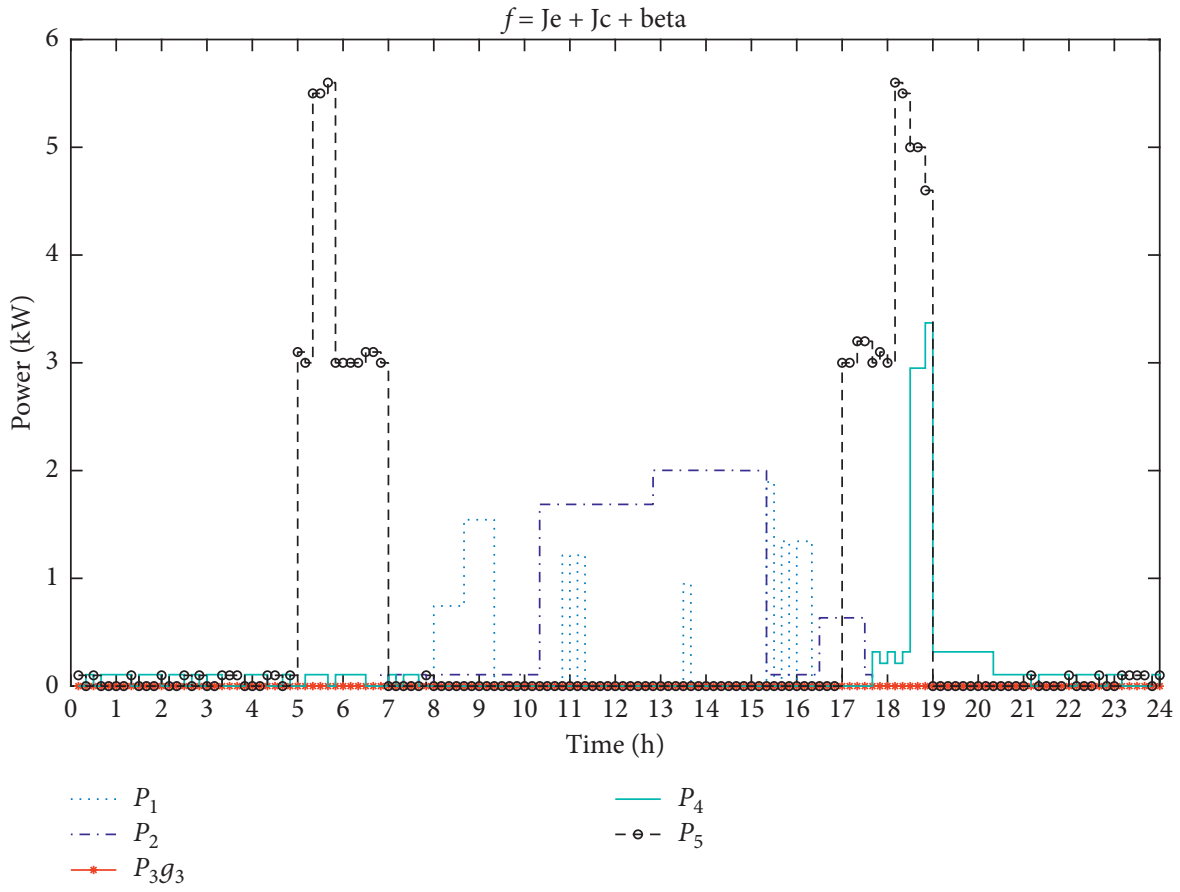

Figure 7: Power dispatch of the new design in case (iii).

From Figures 3, 5, and 7, overlaps between different power supplies can be observed especially during peak hours, while from Figures 4, 6, and 8, none of the time slots allows multiple power supplies. In these cases, the grid power outputs are smoothened under the dispatch with the proposed approach. From the comparative results, supplies become more intermittent because of the contradiction between the renewable penetration objective and the supply constraint. Allowing the combination of multiple power supplies reduces such an intermittent performance while, according to the energy and economy performances in case studies, making better use of the renewable energy sources.

As a conclusion, the proposed approach outperforms the previous model in all cases. When comparing the results of the new and previous designs, it appears that the flexible power dispatch allows more appliances to be scheduled to 


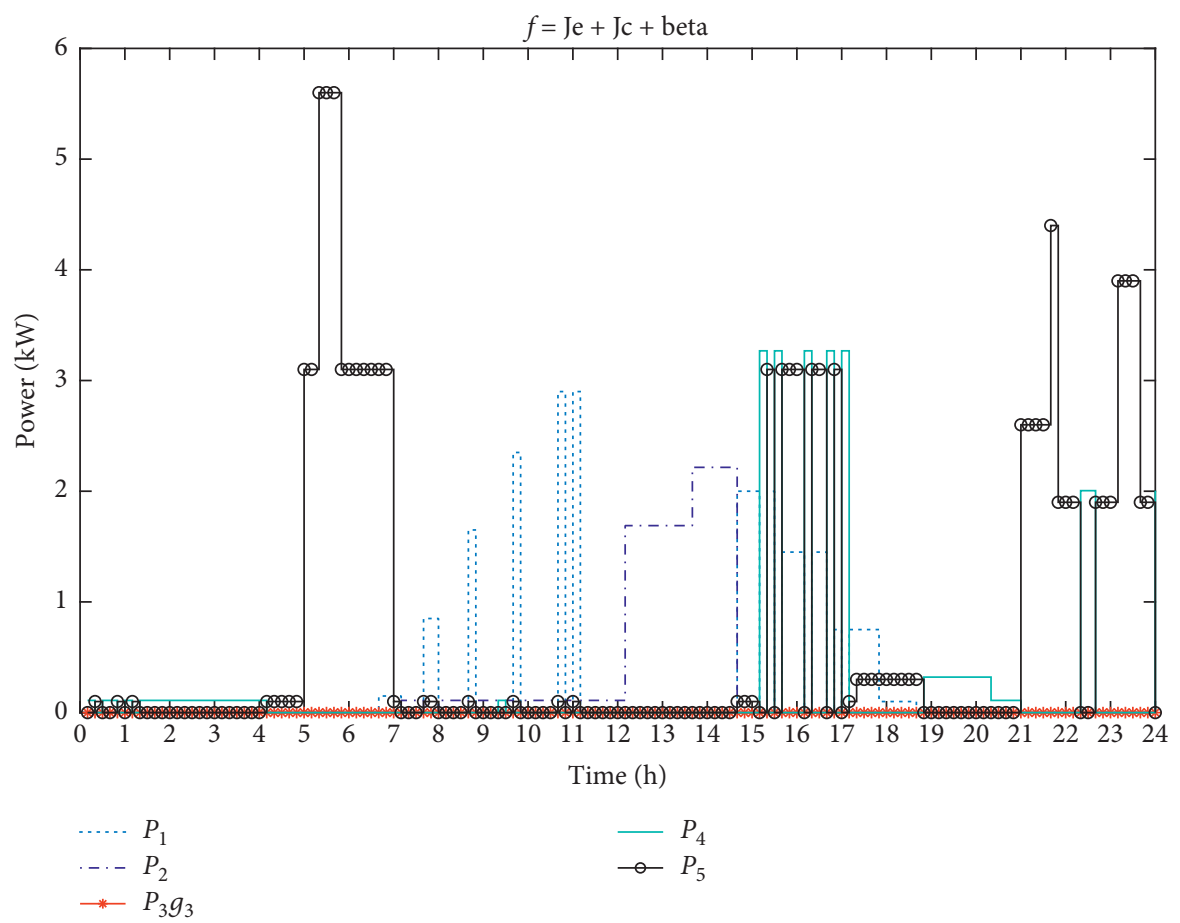

Figure 8: Power dispatch of the previous design in case (iii).

standard and off-peak hours, resulting in a lower energy cost. The objectives $J_{e}$ and $J_{c}$ manifest a certain level of trade-off. This is resulted from the employment of the TOU tariff, where some off-peak hours may be infeasible for the PV system because of its intermittent nature. When comparing the results among the three cases, it can be found that the first two cases can achieve lower energy cost and grid power consumption because of ignoring the inconvenience indicator $\beta$. It invokes an interesting topic for future studies that how to strike a balance between the conflicted interests of user satisfaction and energy efficiency in such a hybrid energy system management.

\section{Conclusions}

This paper investigates a technoeconomic optimization problem for a domestic grid-connected PV-battery hybrid energy system, via extending and improving a previously proposed system design. According to the previous design, the power dispatch is decided for the totality of the electrical loads. In the new model, appliances that comprise the electrical loads are supplied and managed, respectively, via additional power lines and switches from each power source. Furthermore, the appliance time scheduling is incorporated into such a flexible power dispatch. In this way, the system achieves better energy efficiency and economic performances via the technoeconomic optimization. The performances are evaluated by three optimization objectives: minimizing energy cost, maximizing renewable energy penetration, and increasing user satisfaction, over a finite horizon. There are nonlinear objective functions and constraints, as well as discrete and continuous decision variables, in such an optimization problem.
As a result, the problem becomes an MINLP one at a large scale, which is difficult to solve. A competitive swarm optimizer-based numerical solver is thereby designed and employed.

In order to verify that the new design does improve the performances, a case study is investigated, where the power dispatch and appliance time scheduling on a daily basis are applied to a typical South African household hybrid system. There are three optimization cases, each with different objective functions, including only energy cost minimization, only renewable energy penetration maximization, and a weighted sum of the three objectives. Simulations are applied in these cases, where comparative results are also obtained via optimization of the previous system design. The same solver and system configurations are employed. In all cases, the results from the new design outperform results from the previous design. The improvement ranges from $7.1 \%$ to $13.3 \%$ and manifests that further energy efficiency and economic benefits can be achieved by the proposed approach. Furthermore, the solver generally takes around 18.4 minutes to obtain the solution. It verifies that the proposed approach has the potential for application in a real-time context.

There are several future works to investigate based on the current-stage results. Firstly, the performance evaluations, such as the battery wear cost and the renewable energy penetration, are simplified. More practical indicators can be introduced in the future. Secondly, uncertainties from the environment and user demands are inevitable in practice. The real-time feedback mechanism can be introduced to overcome such uncertainties. Thirdly, the game theorybased power dispatch and load scheduling considering conflicted interests call further study. Lastly, the CSO-based 
numerical solver can be further investigated to improve the algorithm performance.

\section{Data Availability}

All relevant data used to support the findings of this study are included within the article.

\section{Conflicts of Interest}

The authors declare that there are no conflicts of interest regarding the publication of this paper.

\section{Acknowledgments}

This work was supported by the National Nature Science Foundation of China under Grant 61803162 and the Fundamental Research Funds for the Central Universities under Grant HUST: 2017 KFYXJJ178.

\section{References}

[1] J. Soares, T. Pinto, F. Lezama, and H. Morais, "Survey on complex optimization and simulation for the new power systems paradigm," Complexity, vol. 2018, Article ID 2340628, 32 pages, 2018.

[2] B. Zhu, H. Tazvinga, and X. Xia, "Switched model predictive control for energy dispatching of a photovoltaic-diesel-battery hybrid power system," IEEE Transactions on Control Systems Technology, vol. 23, pp. 1229-1236, 2014.

[3] S. Shaahid and M. Elhadidy, "Economic analysis of hybrid photovoltaic-diesel-battery power systems for residential loads in hot regions-a step to clean future," Renewable and Sustainable Energy Reviews, vol. 12, pp. 488-503, 2008.

[4] P. Nema, R. Nema, and S. Rangnekar, "A current and future state of art development of hybrid energy system using wind and pv-solar: a review," Renewable and Sustainable Energy Reviews, vol. 13, pp. 2096-2103, 2009.

[5] X. Li, J. Lai, and R. Tang, "A hybrid constraints handling strategy for multiconstrained multiobjective optimization problem of microgrid economical/environmental dispatch," Complexity, vol. 2017, Article ID 6249432, 12 pages, 2017.

[6] T. Cheng, M. Chen, Y. Wang et al., "Adaptive robust method for dynamic economic emission dispatch incorporating renewable energy and energy storage," Complexity, vol. 2018, Article ID 2517987, 13 pages, 2018.

[7] X. Chen, B. Xu, and W. Du, "An improved particle swarm optimization with biogeography-based learning strategy for economic dispatch problems," Complexity, vol. 2018, Article ID 7289674, 15 pages, 2018.

[8] R. Kallel, G. Boukettaya, and L. Krichen, "Demand side management of household appliances in stand-alone hybrid photovoltaic system," Renewable Energy, vol. 81, pp. 123-135, 2015.

[9] S. Phiri and K. Kusakana, "Demand side management of a grid connected pv-wt-battery hybrid system," in Proceedings of the 2016 International Conference on the Industrial and Commercial Use of Energy (ICUE), pp. 45-51, IEEE, Cape Town, South Africa, August 2016.

[10] H. Tazvinga, X. Xia, and J. Zhang, "Minimum cost solution of photovoltaic-diesel-battery hybrid power systems for remote consumers," Solar Energy, vol. 96, pp. 292-299, 2013.
[11] H. Tazvinga, B. Zhu, and X. Xia, "Energy dispatch strategy for a photovoltaic-wind-diesel-battery hybrid power system," Solar Energy, vol. 108, pp. 412-420, 2014.

[12] D. Setlhaolo and X. Xia, "Optimal scheduling of household appliances with a battery storage system and coordination," Energy and Buildings, vol. 94, pp. 61-70, 2015.

[13] S. M. Sichilalu and X. Xia, "Optimal energy control of grid tied pv-diesel-battery hybrid system powering heat pump water heater," Solar Energy, vol. 115, pp. 243-254, 2015.

[14] D. Setlhaolo and X. Xia, "Combined residential demand side management strategies with coordination and economic analysis," International Journal of Electrical Power \& Energy Systems, vol. 79, pp. 150-160, 2016.

[15] F. Yang and X. Xia, "Techno-economic and environmental optimization of a household photovoltaic-battery hybrid power system within demand side management," Renewable Energy, vol. 108, pp. 132-143, 2017.

[16] C. Kagiri, E. M. Wanjiru, L. Zhang, and X. Xia, "Optimized response to electricity time-of-use tariff of a compressed natural gas fuelling station," Applied Energy, vol. 222, pp. 244-256, 2018.

[17] R. Cheng and Y. Jin, "A competitive swarm optimizer for large scale optimization," IEEE Transactions on Cybernetics, vol. 45, pp. 191-204, 2014.

[18] J. Kennedy, "Particle swarm optimization," in Encyclopedia of machine learning, C. Sammut and G. I. Webb, Eds., Springer, Boston, MA, USA, pp. 760-766, 2010.

[19] S. Leva, A. Dolara, F. Grimaccia, M. Mussetta, and E. Ogliari, "Analysis and validation of 24 hours ahead neural network forecasting of photovoltaic output power," Mathematics and Computers in Simulation, vol. 131, pp. 88-100, 2017.

[20] H. Bindner, T. Cronin, P. Lundsager, J. F. Manwell, U. Abdulwahid, and I. Baring-Gould, Lifetime Modelling of Lead Acid Batteries, Forskningscenter Risoe, Roskilde, Denmark, 2005.

[21] C. Wei, M. Benosman, and T. Kim, "Online parameter identification for state of power prediction of lithium-ion batteries in electric vehicles using extremum seeking," International Journal of Control, Automation and Systems, 2019, In press.

[22] C. Yin, X. Huang, S. Dadras et al., "Design of optimal lighting control strategy based on multi-variable fractional-order extremum seeking method," Information Sciences, vol. 465, pp. 38-60, 2018.

[23] F. Cheng, L. Qu, W. Qiao, C. Wei, and L. Hao, "Fault diagnosis of wind turbine gearboxes based on DFIG stator current envelope analysis," IEEE Transactions on Sustainable Energy, vol. 10, no. 3, pp. 1044-1053, 2018.

[24] X. Xia and J. Zhang, "Operation efficiency optimisation modelling and application of model predictive control," IEEE/ CAA Journal of Automatica Sinica, vol. 2, pp. 166-172, 2015.

[25] R. Cheng, "Nature inspired optimization of large problems," Ph.D. thesis, University of Surrey, Guildford, UK, 2016.

[26] Q. Zhao and L.-y. Zhang, "Research on the effect of Dpso in team selection optimization under the background of big data," Complexity, vol. 2018, Article ID 1386407, 14 pages, 2018.

[27] J. Liu, H. Ma, X. Ren, T. Shi, P. Li, and X. Ma, "The continuous-discrete pso algorithm for shape formation problem of multiple agents in two and three dimensional space," Applied Soft Computing, vol. 67, pp. 409-433, 2018.

[28] L. Wang and X.-l. Zheng, "A knowledge-guided multi-objective fruit fly optimization algorithm for the multi-skill resource constrained project scheduling problem," Swarm and Evolutionary Computation, vol. 38, pp. 54-63, 2018. 
[29] H. Tazvinga and T. Hove, Photovoltaic/Diesel/Battery Hybrid Power Supply System, VDM Publishers, Pretoria, South Africa, 2010.

[30] Z. Wu and T. W. Chow, "Neighborhood field for cooperative optimization," Soft Computing, vol. 17, pp. 819-834, 2013.

[31] N. Ao, M. Zhao, Q. Li, S. Qu, and Z. Wu, "Network characteristics for neighborhood field algorithms," Neural Computing and Applications, 2019. 


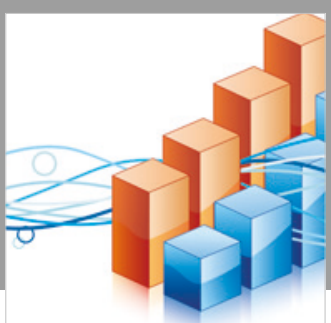

Advances in

Operations Research

\section{-n-m}
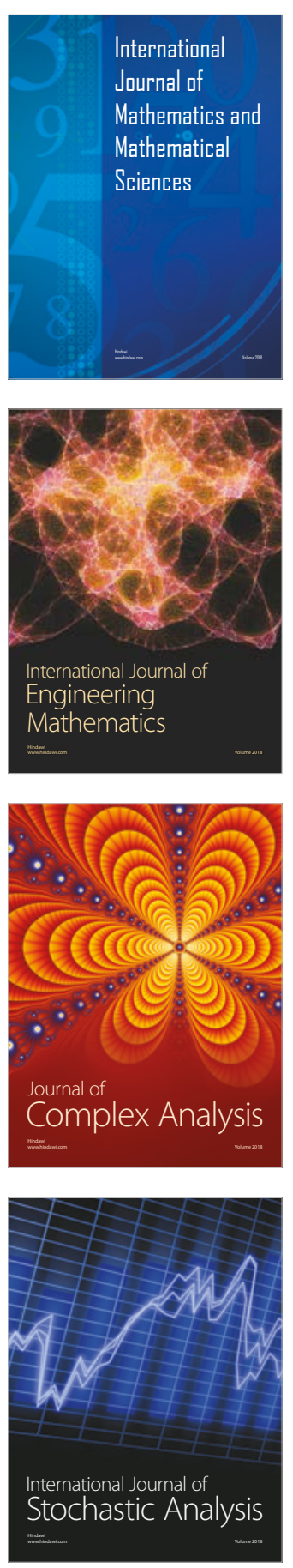
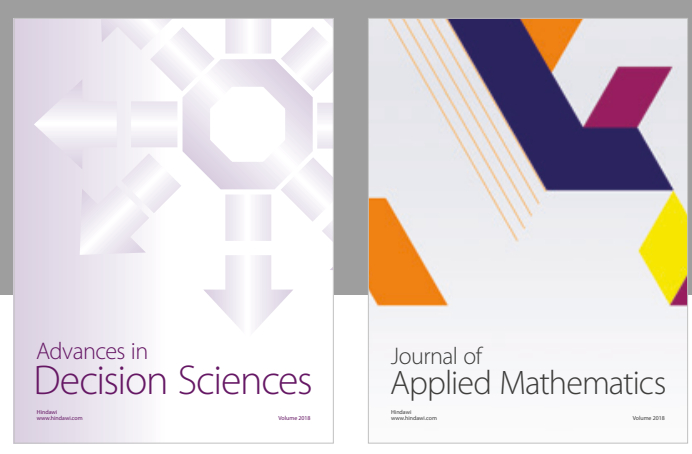

Journal of

Applied Mathematics
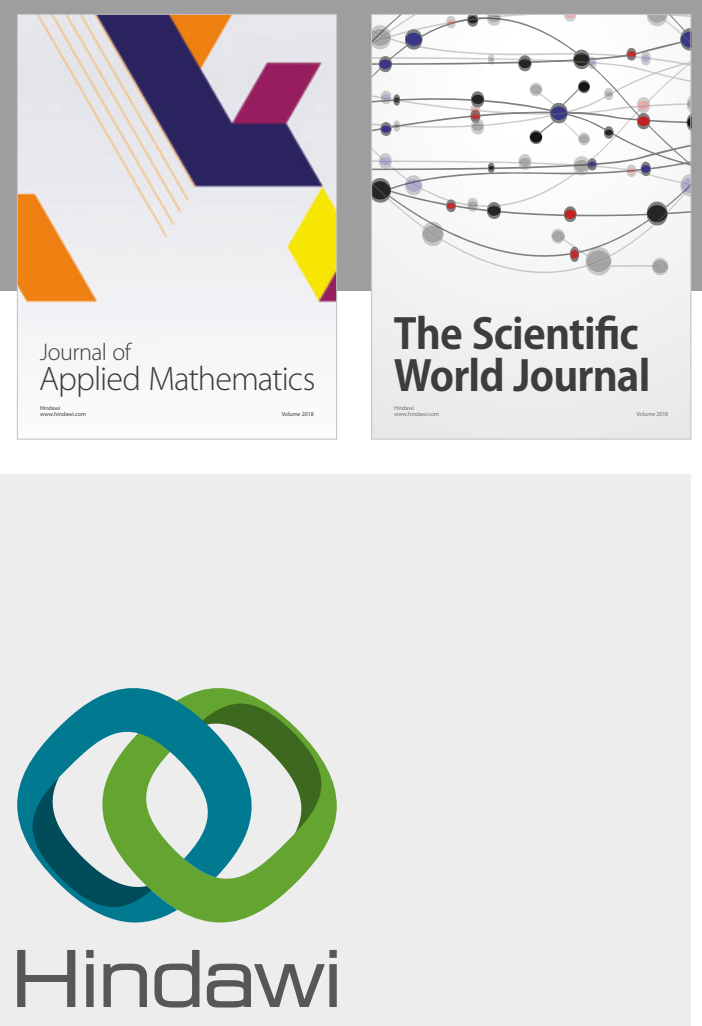

Submit your manuscripts at

www.hindawi.com

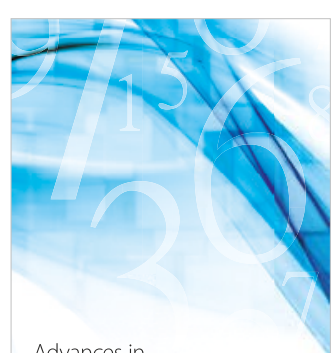

Advances in
Numerical Analysis
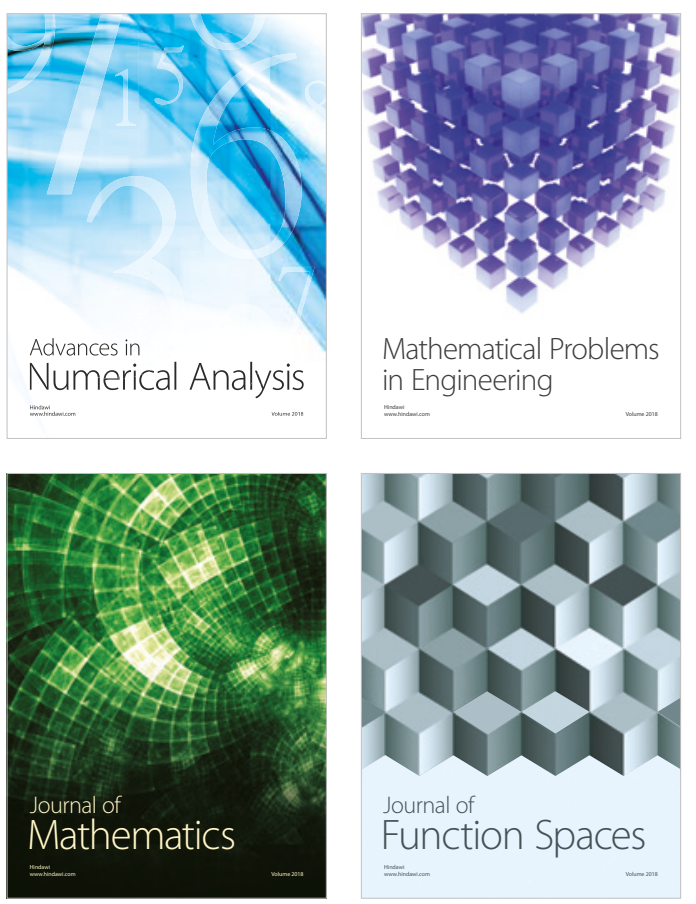

Mathematical Problems in Engineering

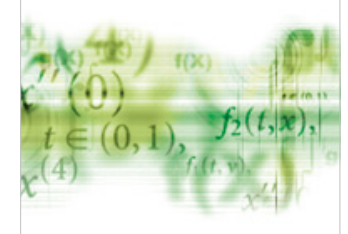

International Journal of

Differential Equations

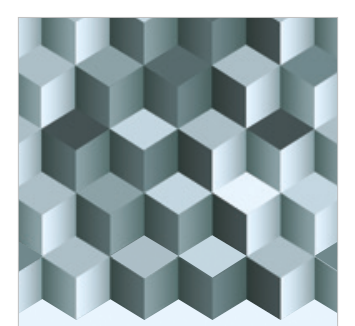

Journal of

Function Spaces
The Scientific

World Journal

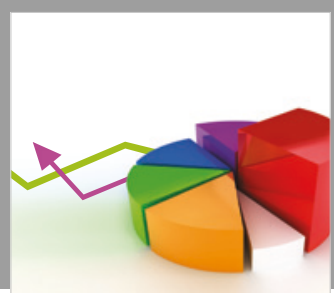

Journal of

Probability and Statistics
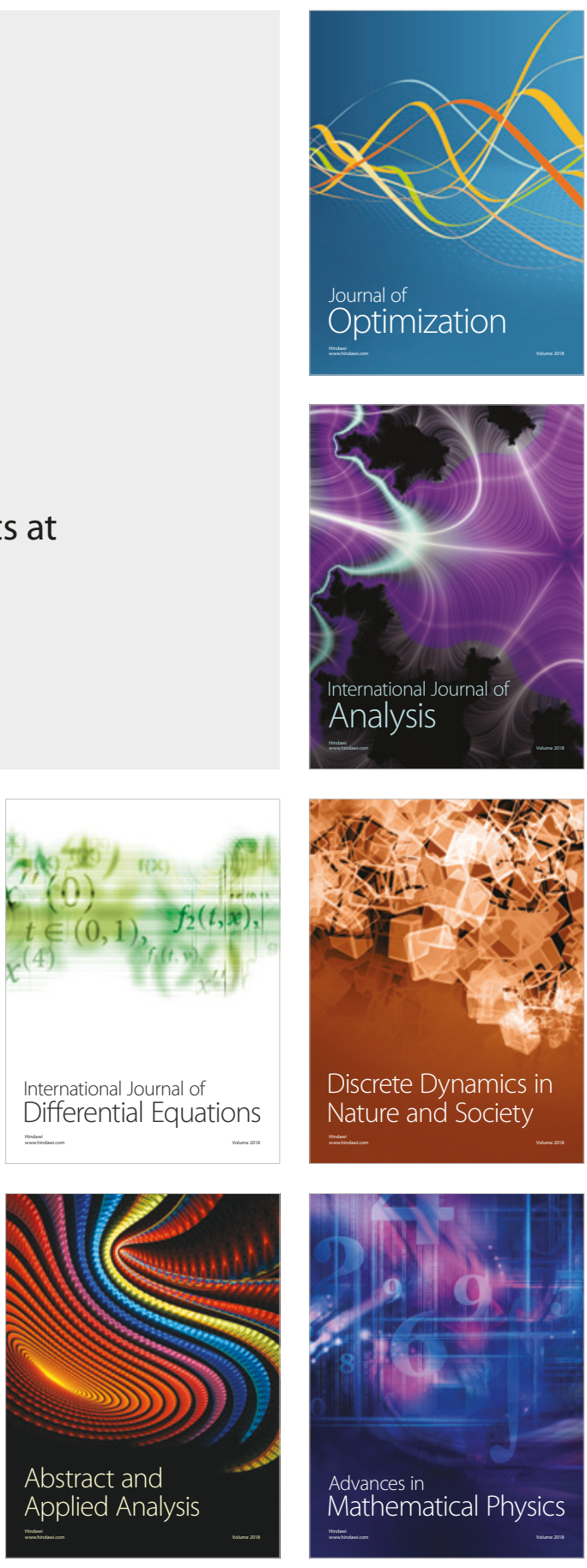